\title{
Dendrobium anosmum Lindl. em áreas verdes na Universidade Federal da Grande Dourados, UFGD
}

\section{Dendrobium anosmum Lindl. in green areas at the Federal University of Grande Dourados, UFGD}

\author{
José Carlos Sorgato \\ Universidade Federal da Grande Dourados (UFGD) \\ E-mail: josesorgato@ufgd.edu.br \\ OrcID: https://orcid.org/0000-0003-0001-5467
}

Luan Marlon Ribeiro

Universidade Federal da Grande Dourados (UFGD)

E-mail: luanmarlon@hotmail.com

OrcID: https://orcid.org/0000-0002-7278-7696

Jackeline Schultz Soares

Universidade Federal da Grande Dourados (UFGD)

E-mail: jacke.schultz@gmail.com

OrcID: https://orcid.org/0000-0001-9497-2975

Rudimara Ferreira Grafen

Universidade Federal da Grande Dourados (UFGD)

E-mail: rudimara55@hotmail.com

OrclD: https://orcid.org/0000-0000

André Luiz Xavier de Araujo

Universidade Federal da Grande Dourados (UFGD)

E-mail: andrexaraujo2016@hotmail.com

OrclD: https://orcid.org/0000-0000

Resumo: Pouco se sabe sobre os ajustes morfofisiológicos de orquídeas germinadas e cultivadas in vitro quando inseridas em áreas verdes. Objetivou-se avaliar a inserção de Dendrobium anosmum Lindl. em áreas verdes na UFGD. Vinte indivíduos foram fixados nos forófitos e submetidos à quatro tratamentos: T1- sem substrato e adubo; T2- com substrato e sem adubo; T3- sem substrato com adubo e T4- com substrato e adubo. Durante 300 dias foi avaliada a sobrevivência (SOB), comprimento (CP) e diâmetro dos pseudobulbos (DP), número de brotos (NB) e de keikis (NK). Houve $100 \%$ de sobrevivência, a menor redução em DP foi observada em T1 e o maior NK em T4. Os maiores valores de CP foram observados em T3 e o maior NB em T2. O fornecimento de adubo e a presença de substrato influenciaram no desenvolvimento de $D$. anosmum quando fixado em forófitos, possibilitando a utilização desta técnica para fins paisagísticos.

Palavras-chave: Floricultura. Orchidaceae. Paisagismo. Plantas ornamentais.

Abstract: There is little knowledge about the morphophysiological adjustments of germinated orchids and cultivated in vitro when inserted in green areas. The objective of this study was to evaluate the insertion of Dendrobium anosmum Lindl. in green areas at the UFGD. Twenty individuals were fixed to the phorophytes and submitted to four treatments: T1- without substrate and fertilizer; T2- with substrate and without fertilizer; T3without substrate with fertilizer and T4- with substrate and fertilizer. For 300 days, survival (SOB), length (CP) and diameter of pseudobulbs (DP), number of shoots (NB) and keikis (NK) were evaluated. There was $100 \%$ survival, the smallest reduction in DP was observed in T1 and the highest NK in T4. The highest CP values were observed in T3 and the highest NB in T2. The supply of fertilizer and the presence of substrate influenced the 
development of $D$. anosmum when fixed in phorophytes, enabling the use of this technique for landscape purposes.

Keywords: Floriculture. Landscaping. Orchidaceae. Ornamental plants.

Data de recebimento: $10 / 08 / 2020$

Data de aprovação: 07/10/2020

DOI: https://doi.org/10.30612/agrarian.v14i51.9615

\section{Introdução}

Orchidaceae é uma das maiores e mais diversificadas famílias dentre as Angiospermas, com cerca de 850 gêneros e 26.567 espécies, sem contar o número de híbridos artificiais, conforme a World Orchid Checklist (Govaerts et al., 2016). Esse grupo tem distribuição cosmopolita, embora não homogênea, com maior abundância de espécies nas regiões tropicais e subtropicais, uma vez que são organismos extremamente especializados que ocupam diversos habitats, comumente apresentando porte herbáceo. De acordo com o habitat podem ser, aquáticas, saprófitas, rupícolas, terrícolas, mas em sua maioria são epífitas, apresentando várias adaptações morfológicas, anatômicas e fisiológicas (Kerbauy, 2011; Flora do Brasil em Construção [FBC], 2020).

Por possuírem diversas formas de vida e elevado potencial ornamental, as orquídeas podem requerer diferentes tratos culturais relacionados às diversas espécies que, ainda podem variar de acordo com o local geográfico no qual as plantas são cultivadas. Deste modo, um desafio no cultivo de orquídeas é entender sua fisiologia, bem como adaptações e estratégias para aquisição e utilização de nutrientes em diferentes condições e tratos culturais (Zhang et al., 2018).

Algumas dessas plantas também podem ser empregadas no paisagismo, incluindo áreas verdes, visto que certos gêneros, híbridos e espécies apresentam rusticidade, florescimento em grande escala e são de fácil cultivo, tais como as do gênero Dendrobium (Mello \& Pantoja, 2014). Dentre essas, destaca-se Dendrobium anosmum Lindl., nativa da Malásia, Laos, Vietnã, Filipinas, Hong Kong e Indonésia. Essa espécie apresenta pseudobulbos pendentes de até $120 \mathrm{~cm}$ de comprimento, com folhas opostas e decíduas. Sua floração tem início no fim da primavera ou no início do verão, apresentando flores com aproximadamente $5 \mathrm{~cm}$ de diâmetro, em diversos tons de rosa e roxo, com interior na cor vinho ou totalmente branco (Araújo, 2017).

O cultivo comercial de orquídeas representa uma atividade importante no mercado mundial de flores e plantas ornamentais, movimentando anualmente bilhões de dólares (Junqueira \& Peetz, 2018). Com a elevada exigência de mudas para suprir o mercado, a utilização de técnicas de cultivo in vitro para diversas espécies de orquídeas já tem seus protocolos estabelecidos, como Cattleya Lindl. (Schneiders, Pescador, Booz, Suzuki, 2012), Dendrobium Sw. (Faria, Rodrigues, Oliveira, Muller, 2004), Cymbidium Sw. (Hossain, Sharma, Pathak, 2009), Vanda (Johnson \& Kane, 2007), que são alguns dos gêneros de maior comercialização (Junqueira \& Peetz, 2018).

Dentre essas técnicas, a germinação assimbiótica tem se mostrado uma ferramenta eficiente para propagação de Orchidaceae, pois resulta em maiores porcentagens de germinação, em relação a condições naturais (Yang et al., 2017), tornando-se importante para produção de mudas a serem introduzidas no ambiente, tanto para fins de conservação ambiental, quanto para o paisagismo.

Ainda, pouco se sabe sobre os ajustes morfofisiológicos de plantas germinadas e cultivadas in vitro quando inseridas em áreas verdes. Assim, conhecer os requerimentos nutricionais e de substratos é importante para definição de protocolos de inserção e cultivo dessas plantas em forófitos. Dessa forma, objetivou-se avaliar a inserção de Dendrobium anosmum Lindl. em áreas verdes na Universidade Federal da Grande Dourados, UFGD.

\section{Material e Métodos}

Plantas de Dendrobium anosmum Lindl. foram fixadas nos forófitos em junho de 2017, em área verde da Universidade Federal da Grande Dourados (UFGD), em Dourados - MS. O clima da região, segundo a classificação de Köppen é do tipo Am (Tropical Monçônico), com verões quentes e invernos secos, temperaturas máximas observadas nos meses de dezembro e janeiro e temperaturas mínimas entre maio e 


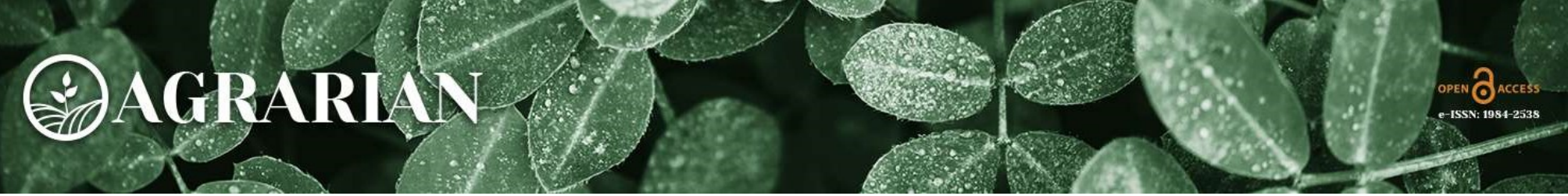

agosto, coincidindo com chuvas excedentes na primavera - verão e déficit hídrico no outono - inverno (Fietz, Fisch, Comunello, Flumignan, 2017).

Para o estudo, foram utilizadas plantas de $D$. anosmum com 18 meses obtidas a partir de germinação assimbiótica e aclimatizadas durante seis meses em viveiro coberto pela sobreposição de duas telas de sombreamento de $50 \%$, (Radiação fotossinteticamente ativa - PAR $=235,1 \mu \mathrm{mol} \mathrm{m}^{-2} \mathrm{~s}^{-1}$ ). Essas sementes foram oriundas de matrizes com seis anos cultivadas no Orquidário da Faculdade de Ciências Agrárias - FCA/UFGD.

Para o experimento foram escolhidos aleatoriamente 20 indivíduos de $D$. anosmum, com cerca de $7 \mathrm{~cm}$ de comprimento. Em seguida, foram fixados em Caesalpinia pluviosa DC. com cordões sintéticos de náilon, na altura de dois metros a partir da base da árvore, na face leste do caule, de forma que a incidência de luz sobre as plantas não fosse direta nos períodos mais quentes do dia, sendo alocada uma orquídea por árvore. Com o intuito de refazer as mesmas práticas do consumidor, que adquire a muda e insere essa orquídea em árvore, não foi realizado nenhum tipo de irrigação durante o período experimental.

Para avaliar a sobrevivência e o ajuste das plantas à nova condição, foram utilizados quatro tratamentos: T1- sem substrato e sem adubo; T2- com substrato e sem adubo; T3- sem substrato com adubo e T4- com substrato e com adubo. Foram pesados $3 \mathrm{~g}$ do adubo NPK 15-11-11 e inserido em trouxas de náilon, sendo fixados acima da planta em uma distância média de $10 \mathrm{~cm}$ para fornecimento lento durante o período experimental. $\mathrm{O}$ substrato utilizado foi composto por esfagno e fibra de coco 1:1 $\mathrm{v} \mathrm{v}^{-1}$ (Figura 1).
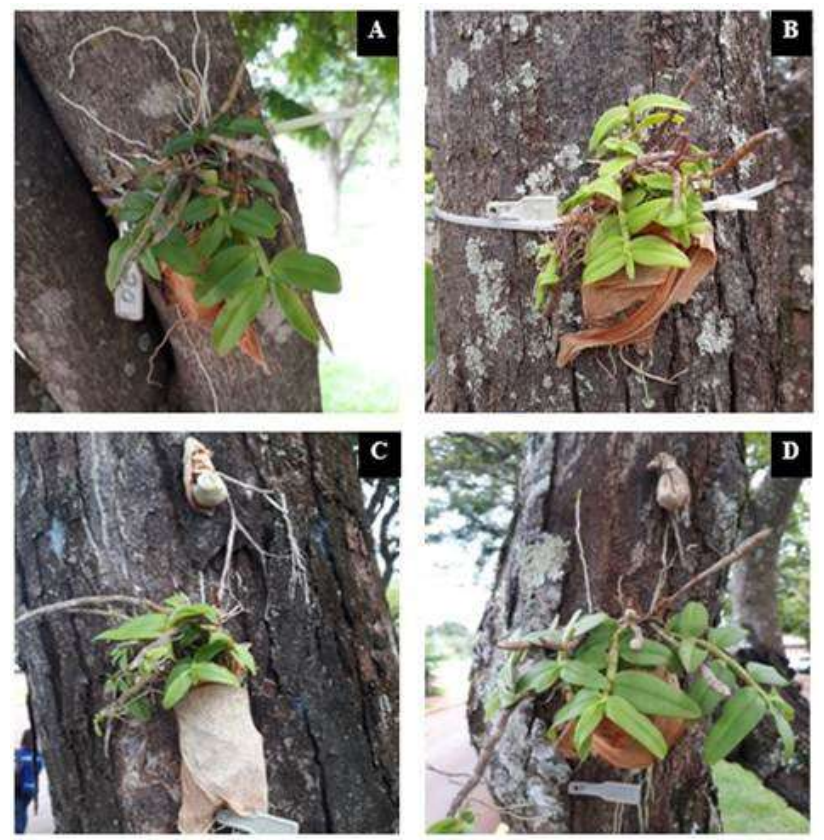

Figura 1. Plantas de Dendrobium anosmum Lindl. (A- T1: sem substrato e sem adubo; B- T2: com substrato e sem adubo; C- T3: sem substrato e com adubo e D- T4: com substrato e com adubo) com 18 meses, introduzidas em áreas verdes.

Mensalmente, durante 300 dias (de julho de 2017 a abril de 2018), foram avaliados o comprimento do pseudobulbo (CP), diâmetro do pseudobulbo (DP), número de brotos (NB) e número de propágulos, denominados (keikis) (NK).

Com intuito de investigar a hipótese de aumento no crescimento das plantas durante a fase ex vitro, de acordo com os tratamentos a que foram inicialmente expostas, foram calculados seus incrementos (I) de DP em relação aos valores iniciais por meio da expressão I = (VF - VI), onde VI é o valor da variável antes da planta ser aclimatizada e VF é o valor da mesma variável após o período ex vitro, sendo seus valores expressos em porcentagem e submetidos à análise de variância.

O delineamento experimental utilizado foi inteiramente casualizado (DIC) em esquema fatorial $10 \times 4$ (dez tempos e quatro tratamentos) com cinco repetições de uma planta cada. Os dados foram transformados para $\sqrt{ }(x+1)$ e, a seguir, submetidos à análise de variância e comparados pelo teste de Scott-Knott $(p<0,05)$ com auxílio do programa SISVAR (Programa de Análises Estatísticas v.5.3. Universidade Federal de Lavras, $M G)$. 


\section{Resultados}

Houve efeito da interação entre tempo e tratamentos $(p<0,05)$ sobre o incremento em diâmetro de pseudobulbos (IDP) e o número de keikis (NK) de $D$. anosmum. A análise também apresentou efeito isolado do tempo $(p<0,05)$ sobre o número de brotos $(N B)$ e dos tratamentos $(p<0,05)$ para todas as caraterísticas avaliadas. Após 300 dias de introdução, as plantas apresentaram porcentagem de sobrevivência de $100 \%$.

De maneira geral, ao longo do período experimental ocorreu redução no diâmetro dos pseudobulbos em todos os tratamentos. Em relação ao tempo de introdução, a análise demonstrou que, para T1 os maiores valores foram observados no período de 30 a 150 dias e para T2 e T3 entre 30 e 90 dias, sem diferença. Para T4 os maiores valores foram encontrados aos 30 dias (redução de $0,62 \%$ ). Ao observar todos os tratamentos, notou-se que a menor redução no IDP ocorreu em T1 (-13,11\%).

Aos 150 dias, plantas que receberam adubação, com ou sem substrato, apresentaram maior redução em diâmetro (T3 $=-33,92 \%$ e T4 $=-36,98 \%)$. O tratamento T1 apresentou a menor redução $(-5,91 \%)$ diferindo dos demais. No entanto, aos 240 dias, mesmo com elevada redução em diâmetro, o T4 apresentou IDP de $30,58 \%$, superior aos demais tratamentos (Tabela 1 ).

Tabela 1. Incremento do diâmetro de pseudobulbo (IDP) de Dendrobium anosmum Lindl.

\begin{tabular}{ccccc}
\hline \multirow{2}{*}{ Tempo (dias) } & \multicolumn{4}{c}{$\ldots \ldots \ldots \ldots \ldots \ldots \ldots \ldots \ldots \ldots \ldots \ldots \ldots \ldots \ldots$} \\
\cline { 2 - 5 } & $-0,51 \mathrm{aA}$ & $-0,82 \mathrm{aA}$ & $-0,22 \mathrm{aA}$ & $-0,62 \mathrm{aA}$ \\
60 & $3,86 \mathrm{aA}$ & $-4,72 \mathrm{aA}$ & $-11,01 \mathrm{aA}$ & $-11,36 \mathrm{bA}$ \\
90 & $12,34 \mathrm{aA}$ & $-5,34 \mathrm{aA}$ & $-17,40 \mathrm{aA}$ & $-14,67 \mathrm{bA}$ \\
120 & $-13,62 \mathrm{aA}$ & $-35,11 \mathrm{cA}$ & $-30,18 \mathrm{bA}$ & $-27,48 \mathrm{cA}$ \\
150 & $-5,91 \mathrm{aA}$ & $-21,36 \mathrm{bA}$ & $-33,92 \mathrm{bB}$ & $-36,98 \mathrm{cB}$ \\
180 & $-23,14 \mathrm{bA}$ & $-37,37 \mathrm{cA}$ & $-35,02 \mathrm{bA}$ & $-38,84 \mathrm{cA}$ \\
210 & $-44,47 \mathrm{cA}$ & $-58,73 \mathrm{dA}$ & $-55,07 \mathrm{cA}$ & $-47,93 \mathrm{cA}$ \\
240 & $-36,76 \mathrm{cB}$ & $-47,84 \mathrm{~dB}$ & $-56,17 \mathrm{cB}$ & $-30,58 \mathrm{cA}$ \\
270 & $-5,14 \mathrm{aA}$ & $-18,07 \mathrm{bA}$ & $-34,80 \mathrm{bB}$ & $-19,21 \mathrm{bA}$ \\
300 & $-17,74 \mathrm{bA}$ & $-37,99 \mathrm{cA}$ & $-36,34 \mathrm{bA}$ & $-33,47 \mathrm{cA}$ \\
Média & $-13,11$ & $-26,74$ & $-31,01$ & $-26,12$ \\
C.V. $\%)$ & & & 6,56 & \\
\hline
\end{tabular}

Médias seguidas de mesma letra minúscula na coluna e maiúscula na linha em cada variável, não diferem estatisticamente entre si, pelo teste de Scott-Knott $(p<0,05)$. T1= sem substrato e sem adubo; T2= com substrato e sem adubo; T3= sem substrato e com adubo e T4= com substrato e com adubo e tempo (dias).

Os keikis começaram a surgir a partir dos 210 dias após a introdução em todos os tratamentos. O maior valor de NK $(5,00)$ foi observado no T4, diferindo dos demais tratamentos. Desse período em diante, de modo geral, houve mortalidade dos keikis (Tabela 2).

Tabela 2. Valores médios de número de keikis (NK) de Dendrobium anosmum Lindl.

\begin{tabular}{ccccc}
\hline \multirow{2}{*}{ Tempo (dias) } & \multicolumn{4}{c}{$\ldots \ldots \ldots \ldots \ldots \ldots \ldots \ldots \ldots \ldots \ldots \ldots \ldots \ldots \ldots \ldots \ldots \ldots \ldots \ldots \ldots \ldots \ldots \ldots \ldots \ldots \ldots$} \\
\cline { 2 - 5 } & T1 & T2 & T3 & T4 \\
\hline 30 & $0,00 \mathrm{aA}$ & $0,00 \mathrm{bA}$ & $0,00 \mathrm{cA}$ & $0,00 \mathrm{cA}$ \\
90 & $0,00 \mathrm{aA}$ & $0,00 \mathrm{bA}$ & $0,00 \mathrm{cA}$ & $0,00 \mathrm{cA}$ \\
120 & $0,00 \mathrm{aA}$ & $0,00 \mathrm{bA}$ & $0,00 \mathrm{cA}$ & $0,00 \mathrm{cA}$ \\
150 & $0,00 \mathrm{aA}$ & $0,00 \mathrm{bA}$ & $0,00 \mathrm{cA}$ & $0,00 \mathrm{cA}$ \\
180 & $0,00 \mathrm{aA}$ & $0,00 \mathrm{bA}$ & $0,00 \mathrm{cA}$ & $0,00 \mathrm{cA}$ \\
210 & $0,00 \mathrm{aA}$ & $0,00 \mathrm{bA}$ & $0,00 \mathrm{cA}$ & $0,00 \mathrm{cA}$ \\
240 & $1,00 \mathrm{aD}$ & $2,20 \mathrm{aC}$ & $3,69 \mathrm{aB}$ & $5,00 \mathrm{aA}$ \\
270 & $1,00 \mathrm{aC}$ & $1,50 \mathrm{aC}$ & $3,60 \mathrm{aA}$ & $2,47 \mathrm{bB}$ \\
30 & $0,00 \mathrm{aB}$ & $0,50 \mathrm{bB}$ & $2,00 \mathrm{bA}$ & $0,45 \mathrm{cB}$ \\
Média & $0,00 \mathrm{aB}$ & $0,87 \mathrm{bB}$ & $3,40 \mathrm{aA}$ & $0,47 \mathrm{cB}$ \\
C.V. (\%) & 0,20 & 0,51 & 1,27 & 0,84 \\
\hline
\end{tabular}


Médias seguidas de mesma letra minúscula na coluna e maiúscula na linha em cada variável, não diferem estatisticamente entre si, pelo teste de Scott-Knott $(p<0,05)$. T1= sem substrato e sem adubo; T2= com substrato e sem adubo; T3= sem substrato e com adubo e T4= com substrato e com adubo.

Aos 210 dias, ao mesmo tempo em que foi observado maior NK, também houve maior redução em IDP das plantas submetidas ao T4. Nesse mesmo período foram observadas maiores médias de precipitação e umidade relativa do ar (Figura 2).

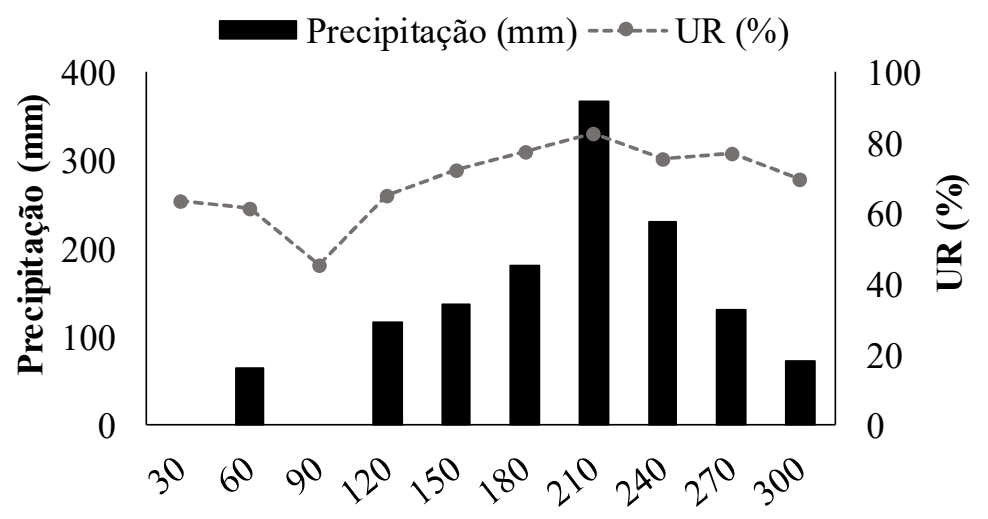

Dias após a introdução

Figura 2. Valores médios de precipitação $(\mathrm{mm})$ e umidade relativa do ar (\%UR), durante o período de introdução de Dendrobium anosmum Lindl. em áreas verdes.

Quanto ao efeito isolado dos tratamentos, plantas sem substrato e que receberam adubação apresentaram maiores valores de comprimento de pseudobulbos $(\mathrm{CP})(\mathrm{T} 3=81,92 \mathrm{~mm})$. Entretanto, aquelas que não receberam adubação, mas com a presença de substrato apresentaram maior NB $(T 2=5,36)$ (Figura $3)$.
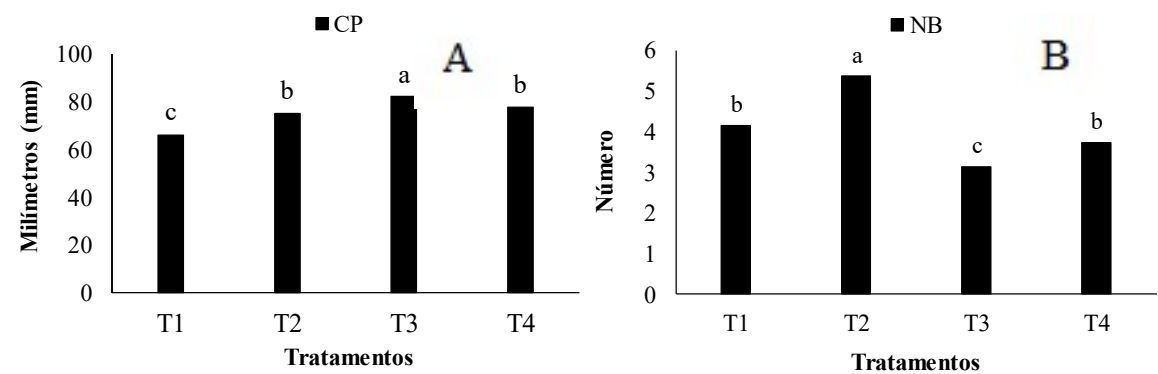

Figura 3. A: comprimento de pseudobulbos (CP) e B: número de brotos (NB) de Dendrobium anosmum Lindl. em função dos tratamentos: T1- sem substrato e sem adubo; T2- com substrato e sem adubo; T3sem substrato e com adubo e T4- com substrato e com adubo.

Ao se observar os resultados do efeito isolado do tempo sobre o NB, notou-se aumento de brotos no decorrer do período experimental até os 210 dias (6,58 brotos). No entanto, aos 240 dias houve diminuição (5,32 brotos) no NB e aos 270 e 300 dias os valores voltaram a aumentar (6,86 e 6,77 brotos respectivamente) (Figura 4). 


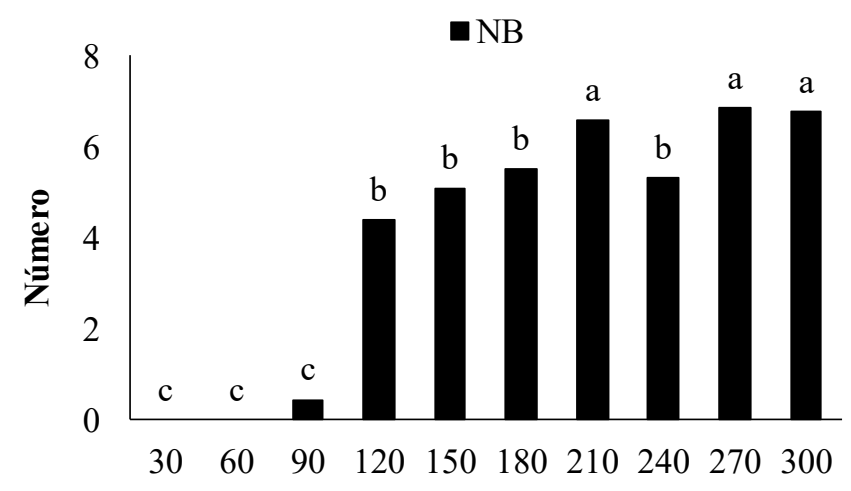

Tempo (dias)

Figura 4. Número de brotos (NB) de Dendrobium anosmum Lindl. após o tempo (dias) de introdução em áreas verdes.

\section{Discussão}

De maneira geral as plantas de $D$. anosmum fixadas em forófitos de Caesalpinia pluviosa apresentaram elevada porcentagem de sobrevivência, mesmo sendo inseridas na época seca (0,0 mm de chuvas e $60 \%$ UR) e tendo seu diâmetro de pseudobulbos diminuído em todos os tratamentos. Aos 210 dias houve a formação de keikis e novas brotações. Já o comprimento dos pseudobulbos aumentou em detrimento do número de brotos nos tratamentos com adubo.

A elevada porcentagem de sobrevivência apresentada neste estudo pode estar associada ao metabolismo crassuláceo apresentado por espécies do gênero Dendrobium. Os indivíduos desse gênero são todos epifíticos e bastante flexíveis quanto às vias fotossintéticas que utilizam, variando entre as vias C3 e CAM (Winter \& Ziegler, 1992; Rodrigues et al., 2013; Qiu, Sultana, Liu, Yin, Wang, 2015). Lüttge (2004) afirma que algumas espécies podem apresentar metabolismo $\mathrm{C} 3 \mathrm{em}$ ótimas condições ambientais e mudar naturalmente para a via CAM em condições de estresse, metabolismo este que oferece eficiente custo/benefício em relação ao consumo de água e absorção de $\mathrm{CO}_{2}$ (Silvera et al., 2010), aumentando assim a sobrevivência dessas plantas.

Como as plantas foram fixadas em período de baixa pluviosidade, apresentaram redução no diâmetro dos pseudobulbos. Para plantas epífitas, a condição de déficit hídrico é ainda mais evidente se considerado que o seu forófito possui limitada capacidade de reter água (Stancato, Mazzafera, Buckeridge, 2001; He, Norhafis, Qin, 2013). Ainda, em condição de estresse por déficit hídrico, há uma mobilização de açúcares em direção aos órgãos com maior demanda energética. Nas orquídeas, esta mobilização pode ocorrer a partir dos pseudobulbos em direção às folhas, quando estas estão com uma demanda energética maior do que sua produção (Stancato et al., 2001; Zampirollo, 2016), o que justifica a perda em diâmetro dessas estruturas de reserva.

Quanto ao número de keikis, de modo geral, a partir do início da estação chuvosa, os tratamentos que receberam adubação (T3 e T4) apresentaram maiores valores. Segundo Sorgato, Soares, Pinto, Rosa (2015), a emissão de keikis é uma resposta fisiológica da planta que, em condições de estresse, emite novas estruturas vegetativas como forma de perpetuação da espécie. Sendo assim, podemos inferir que no início do período das chuvas, as plantas que receberam adubação provavelmente direcionaram os fotoassimilados provenientes do fornecimento nutricional para uma maior expressão de keikis.

Já para comprimento do pseudobulbo e número de brotos, segundo Tejeda-Sartorius, Trejo-Tèllez, RiosBarreto, Rodríguez-de La (2018) maior número e comprimento de pseudobulbos são indicadores indiretos do bom estado nutricional da planta. Sendo assim, pode-se observar que plantas que receberam adubação, mas não foram fixadas com substrato investiram suas reservas nutricionais no aumento do comprimento dos pseudobulbos em detrimento do número de brotações. Essa característica é importante para o $D$. anosmum, uma vez que seu florescimento acorre ao longo dos pseudobulbos.

Até os 90 dias de introdução, de julho a setembro de 2017, a precipitação e a \%UR, em geral, foram menores. Esse período sem chuvas se torna um fator limitante para as plantas, seja do ponto de vista comercial (no caso das culturas agrícolas) ou do ponto de vista conservacionista (no caso das plantas nativas) (Han \& Wagner, 2014). Ainda, as respostas ao déficit hídrico podem variar de maneira complexa de acordo com a 
espécies vegetais, uma vez que algumas apresentam alterações adaptativas que as permitem tolerar estas condições, enquanto outras simplesmente sucumbem ao fator de estresse (Zampirollo, 2016).

Os resultados encontrados neste trabalho evidenciam que, no período de maiores precipitações e maior \%UR (aos 210 dias), ocorreu também a menor redução em diâmetro dos pseudobulbos e intensificação da formação de brotações e keikis. De acordo com Soares (2018), a presença de substrato e adubação junto ao clima favorável pode alterar a temperatura da planta, que está relacionada tanto à temperatura do meio quanto ao seu estado hídrico, podendo favorecer seu desenvolvimento e elevando os valores das variáveis analisadas.

Em períodos de menor precipitação, as plantas epífitas enfrentam déficit hídrico, já que a casca do forófito onde estão inseridas possui limitada capacidade de reter água e, ainda são expostas a radiação solar vinda do dossel (He et al., 2013). Sendo assim, a baixa disponibilidade de água atinge diretamente a abertura estomática e a estrutura dos cloroplastos, levando ao decréscimo nas taxas de transpiração e assimilação de $\mathrm{CO}_{2}$, o que por sua vez, afeta a distribuição, mobilização e acúmulo de fotoassimilados (Waseem et al., 2011).

Para a inserção de espécies de orquídeas no paisagismo em áreas verdes há a necessidade de investimentos em pesquisas, para que assim possam existir técnicas e metodologias que contribuam para esse fim. Diante dos resultados apresentados, pode se inferir que $D$. anosmum apresenta características que permitem seu desenvolvimento frente às limitações climáticas do ambiente.

\section{Conclusão}

O fornecimento de adubo e a presença de substrato influenciaram positivamente no desenvolvimento das plantas de $D$. anosmum, possibilitando a fixação dessas plantas em forófitos para fins paisagísticos em áreas verdes, mesmo sendo inseridas em época de baixa pluviosidade e umidade relativa do ar.

\section{Referências}

Araújo, R. (2017). Orquídeas Dendrobium. São Paulo: Editora Europa.

Faria, R. T., Rodrigues, F. N., Oliveira, L. V. R., Müller, C. (2004). In vitro Dendrobium nobile plant growth and rooting in different sucrose concentrations. Horticultura Brasileira, 22(4), 780-783. http://dx.doi.org/10.1590/S0102-05362004000400023

Fietz, C. R., Fisch, G. F., Comunello, E., Flumignan, D. L. (2017). O clima da região de Dourados, MS. Dourados: Embrapa Agropecuária Oeste, 34p. (Documentos Embrapa, n.138).

Flora do Brasil em Construção. (2020). Lista de espécies da flora do Brasil. Disponível em: <http://floradobrasil.jbrj.gov.br/>. Acesso em: 14/07/2020.

Govaerts, R., Bernet, P., Kratochvil, K., Gerlach, G., Carr, G., Alrich, P., Pridgeon, A. M., Pfahl, J., Campacci, M. A., Holland Baptista, D., Tigges, H., Shaw, J., Cribb, P. J., George, A., Kreuz, K., Wood, J. J. (2016). World checklist of selected plant families (WCSP). Disponível em: http://apps.kew.org/wcsp/namedetail.do?name_id=109178. Acesso em: 23/03/2019.

Han, S. K., Wagner, D. (2014). Role of chromatin in water stress responses in plants. Journal of Experimental Botany, 65(10), 2785-2799. 10.1093/jxb/ert403

He, J., Norhafis, H., Qin, L. (2013). Responses of green leaves and green pseudobulbs of CAM Orchid Cattleya laeliocattleya Aloha Case to drought stress. Journal of Botany, 2013(1), 01-09. https://doi.org/10.1155/2013/710539

Hossain, M. M., Sharma, M., Pathak, P. (2009). Cost effective protocol for in vitro mass propagation of Cymbidium aloifolium (L.) Sw. a medicinally important orchid. Engineering in Life Sciences, 9(6), 444-453. https://doi.org/10.1002/elsc.200900015

Junqueira, A. H., Peetz, M. S. (2018). Sustainability in Brazilian floriculture: introductory notes to a systemic approach. Ornamental Horticulture, 24(2), 155-162. http://dx.doi.org/10.14295/oh.v24i2.1253

Johnson, T. R., Kane, M. E. (2007). Asymbiotic germination of ornamental Vanda: in vitro germination and development of three hybrids. Plant Cell, Tissue and Organ Culture, 91(3), 251-261. 10.1007/s11240-007-9291$\underline{7}$ 
Kerbauy, G. B. (2011). Micropropagação comercial de orquídeas: conquistas, desafios e perspectivas. In: Gerald, L.T.S. (Ed.). Biofábrica de plantas: produção industrial de plantas in vitro. São Paulo: Antiqua, 245-383.

Lüttge, U. (2004). Ecophysiology of Crassulacean Acid Metabolism (CAM). Annals of Botany, 93(6), 629-652. 10.1093/aob/mch087

Mello, A. C. F., Pantoja, S. C. S. (2014). Orquídeas no paisagismo da área urbana de Ipanema-RJ. Revista Eletrônica Novo Enfoque, 18(18), 87-108.

Qiu, S., Sultana, S., Liu, Z. D., Yin, L. Y., Wang, C. Y. (2015). Identification of obligate C3 photosynthesis in Dendrobium. Photosynthetica, 53(2), 168-176. 10.1007/s11099-015-0110-9

Rodrigues, M. A., Matiz, A., Cruz, A. B., Matsumura, A. T., Takahashi, C. A., Hamachi, L., Félix, L. M., Pereira, P. N., Latansio-Aidar, S. P., Aidar, M. P. M., Demarco, D., Freschi, L., Mercier, H. (2013). Spatial patterns of photosynthesis in thin- and thick leaved epiphytic orchids: unravelling C3-CAM plasticity in an organcompartmented way. Annals of Botany, 112(1), 17-29. https://doi.org/10.1093/aob/mct090

Schneiders, D., Pescador, R., Booz, M. R., Suzuki, R. M. (2012). Germinação, crescimento e desenvolvimento in vitro de orquídeas (Cattleya spp., Orchidaceae). Revista Ceres, 59(2), 185-191. http://dx.doi.org/10.1590/S0034-737X2012000200006

Silvera, K., Neubig, K. M., Whitthen, W. M., Williams, M. H., Winter, K., Cushman, J. C. (2010). Evolution along the crassulacean acid metabolism continuum. Functional Plant Biology, 37(1), 995-1010. https://doi.org/10.1071/FP10084

Soares, J. S. (2018). O cultivo in vitro como alternativa para a conservação de Schomburgkia crispa Lindl. (Orchidaceae) no bioma Cerrado. 81f. Tese (Doutorado em Agronomia) - Universidade Estadual de Mato Grosso do Sul - Campus de Dourados - MS.

Sorgato, J. C., Soares, J. S., Pinto, J. V. D. C., Rosa, Y. B. C. J. (2015). Potencial germinativo de sementes e qualidade de keikis de Dendrobium nobile em diferentes fases do desenvolvimento dos frutos. Ciência Rural, 45(11), 1965-1971. https://doi.org/10.1590/0103-8478cr20141129

Stancato, G. C., Mazzafera, P., Buckeridge, M. S. (2001). Effect of a drought period on the mobilisation of nonstructural carbohydrates, photosynthetic efficiency and water status in an epiphytic orchid. Plant Physiology and Biochemistry, 39(1), 1009-1016. https://doi.org/10.1016/S0981-9428(01)01321-3

Tejeda-Sartorius, O., Trejo-Téllez, L. I., Ríos-Barreto, Y., Rodríguez-De La O, J. L. (2018). Mineral fertilization and biofertilization in physiological parameters of the orchid Laelia anceps subsp. anceps. Revista Chapingo, Serie Horticultura, 24(3), 181-190. https://doi.org/10.5154/r.rchsh.2017.07.027

Waseem, M., Ali, A., Tahir, M., Nadeem, M. A., Ayub, M., Tanveer, A., Ahmad, R., Hussain, M. (2011). Mechanisms of drought tolerance in plant and its management through different methods. Continental Journal of Agricultural Science, 5(1), 10-25.

Winter, K., Ziegler, H. (1992). Induction of crassulacean acid metabolism in Mesembryanthemum crystallinum increases reproductive success under conditions of drought and salinity stress. Oecologia, 92(4), 475-479. 10.1007/BF00317838

Yang, F., Sun, A., Zhu, J., Downing, J., Song, X., Liu, H. (2017). Impacts of host trees and sowing conditions on germination success and a simple ex situ approach to generate symbiotic seedlings of a rare epiphytic Orchid endemic to Hainan Island, China. Botanical Review, 83(10), 74-86. 10.1007/s12229-017-9178-1

Zampirollo, J. B. (2016). Atividade fotossintética em folhas e pseudobulbos de Cattleya warneri (CAM) e Miltonia spectabilis $\left(C_{3}\right)$ sob déficit hídrico. 106 f. Dissertação (Mestrado em Biodiversidade Tropical) - Universidade Federal do Espírito Santo - Campus do Espírito Santo - ES.

Zhang, S., Yang, Y., Li, J., Qin, J., Zhang, W., Huang, W., Hu, H. (2018). Physiological diversity of orchids. Plant diversity, 40(4), 196-208. https://doi.org/10.1016/i.pld.2018.06.003 